\title{
Carbene- and Carbyne-like Behavior of the Mo-P Multiple Bond in a Dimolybdenum Complex Inducing Trigonal Pyramidal Coordination of a Phosphinidene Ligand.
}

\author{
M. Angeles Alvarez, Inmaculada Amor, M. Esther García, Daniel García-Vivó and Miguel A. Ruiz* \\ Departamento de Química Orgánica e Inorgánica/IUQOEM, Universidad de Oviedo, 33071 Oviedo, Spain.
}

\section{Supporting Information}

\section{Preparative Procedures and Data for New Compounds.}

\section{General Procedures and Starting Materials}

All manipulations and reactions were carried out under a nitrogen (99.9990\%) atmosphere using standard Schlenk techniques. Solvents were purified according to literature procedures, ${ }^{1}$ and distilled prior to use. Petroleum ether refers to that fraction distilling in the range 65-70 ${ }^{\circ} \mathrm{C}$. Compound $\left[\mathrm{Mo}_{2} \mathrm{Cp}\left(\mu-\kappa^{1}: \kappa^{1}, \eta^{5}-\mathrm{PC}_{5} \mathrm{H}_{4}\right)(\mathrm{CO})_{2}\left(\eta^{6}-\mathrm{HR} *\right)\right](2)\left(\mathrm{Cp}=\eta^{5}-\right.$ $\left.\mathrm{C}_{5} \mathrm{H}_{5} ; \mathrm{HR}^{*}=1,3,5-\mathrm{C}_{6} \mathrm{H}_{3}{ }^{t} \mathrm{Bu}_{3}\right)$ was prepared in situ as described previously. ${ }^{2}$ All other reagents were obtained from the usual commercial suppliers and used as received. Chromatographic separations were carried out using jacketed columns. Commercial alumina (activity I, 150 mesh) was degassed under vacuum prior to use. The latter was mixed under nitrogen with the appropriate amount of water to reach the activity desired. IR C-O stretching frequencies were measured in solution, are given in $\mathrm{cm}^{-1}$ and are referred to as $v(\mathrm{CO})$ (solvent). Nuclear Magnetic Resonance (NMR) spectra were routinely recorded at $400.13\left({ }^{1} \mathrm{H}\right)$, $161.97\left({ }^{31} \mathrm{P}\left\{{ }^{1} \mathrm{H}\right\}\right)$ or $100.63 \mathrm{MHz}\left({ }^{13} \mathrm{C}\left\{{ }^{1} \mathrm{H}\right\}\right)$ at $290 \mathrm{~K}$ in $\mathrm{CD}_{2} \mathrm{Cl}_{2}$ solutions unless otherwise stated. Chemical shifts $(\delta)$ are given in ppm, relative to internal tetramethylsilane $\left({ }^{1} \mathrm{H},{ }^{13} \mathrm{C}\right)$ or external $85 \%$ aqueous $\mathrm{H}_{3} \mathrm{PO}_{4}$ solutions $\left({ }^{31} \mathrm{P}\right)$. Coupling constants $(J)$ are given in Hertz.

Preparative Procedures, Spectroscopic and Microanalytical data for the New Compounds.

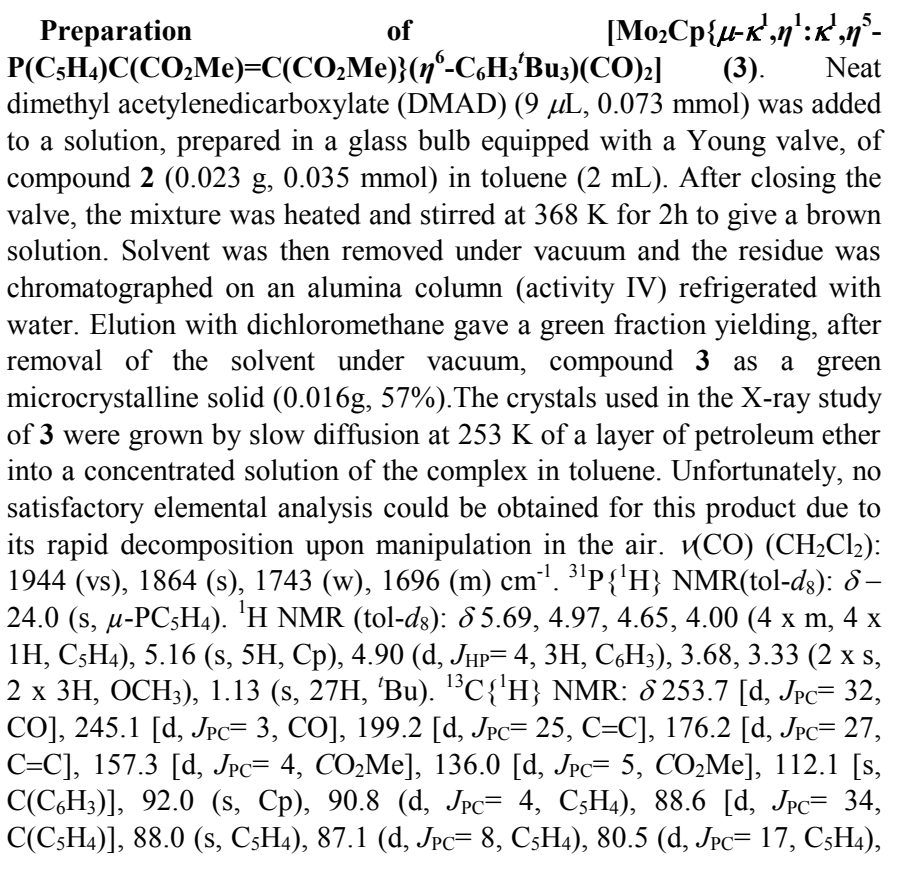

$71.7\left(\mathrm{~s}, \mathrm{C}_{6} \mathrm{H}_{3}\right), 51.3,51.0\left(2 \times \mathrm{s}, 2 \times \mathrm{OCH}_{3}\right), 34.5\left[\mathrm{~s}, \mathrm{C}\left({ }^{t} \mathrm{Bu}\right)\right], 31.3[\mathrm{~s}$, $\left.\mathrm{CH}_{3}\right]$.

Preparation of $\left[\mathrm{Mo}_{2} \mathrm{CpCl}\left\{\mu-\kappa^{1}: \boldsymbol{K}^{1}, \eta^{5}-\mathrm{P}(\mathrm{H}) \mathrm{C}_{5} \mathrm{H}_{4}\right\}\left(\eta^{6}-\right.\right.$ $\left.\mathbf{C}_{6} \mathbf{H}_{3}{ }^{t} \mathbf{B} \mathbf{u}_{3}\right)(\mathbf{C O})_{2}$ ] (4). Hydrogen chloride $(15 \mu \mathrm{L}$ of a $4 \mathrm{M}$ solution in diethylether, $0.060 \mathrm{mmol}$ ) was added to a solution, prepared in a glass bulb equipped with a Young valve, of compound 2 (0.036 g, 0.055 $\mathrm{mmol})$ in dichloromethane $(3 \mathrm{~mL})$, and the mixture was stirred for $3.5 \mathrm{~h}$ to give a dark-red solution. The solvent was then removed under vacuum, the residue was extracted with petroleum ether $(15 \mathrm{~mL})$, and the extract filtered using a cannula to give a red-violet solution. Removal of the solvent from the latter solution under vacuum yielded compound 4 as a violet microcrystalline solid ( $0.020 \mathrm{~g} 53 \%)$. Compound 4 reverts to the starting complex 2 upon treatment with strong bases such as DBU $(1,8$ diazabicyclo [5.4.0] undec-7-ene) or during chromatography on alumina (activity IV). Anal. Calcd for $\mathrm{C}_{30} \mathrm{H}_{40} \mathrm{Mo}_{2} \mathrm{O}_{2} \mathrm{ClP}: \mathrm{C}, 52.15 ; \mathrm{H}, 5.84$. Found: C, 51.78; H, 5.45. v(CO) (petroleum ether): 1969 (vs), 1885 (d), $1874(\mathrm{~m}) . N M R$ data for the major isomer: ${ }^{31} \mathrm{P}$ NMR: $\delta-14.5\left(\mathrm{~d}, J_{\mathrm{PH}}=\right.$ 290, $\mu$ - $\left.\mathrm{PHC}_{5} \mathrm{H}_{4}\right) .{ }^{1} \mathrm{H}$ NMR: $\delta 5.43$ (s, 5H, Cp) 5.18, 5.15, (2 x m, br, $2 \mathrm{x}$ $\left.1 \mathrm{H}, \mathrm{C}_{5} \mathrm{H}_{4}\right), 4.58\left(\mathrm{~m}, \mathrm{br}, 2 \mathrm{H}, \mathrm{C}_{5} \mathrm{H}_{4}\right), 4.90\left(\mathrm{~d}, J_{\mathrm{HP}}=4,3 \mathrm{H}, \mathrm{C}_{6} \mathrm{H}_{3}\right), 3.72(\mathrm{dd}$, $\left.J_{\mathrm{PH}}=290, J_{\mathrm{HH}}=1,1 \mathrm{H}, \mathrm{H}-\mathrm{P}\right), 1.18\left(\mathrm{~s}, 27 \mathrm{H},{ }^{t} \mathrm{Bu}\right) .{ }^{13} \mathrm{C}\left\{{ }^{1} \mathrm{H}\right\} \mathrm{NMR}: \delta 259.4$ [d, $\left.J_{\mathrm{PC}}=30, \mathrm{CO}\right], 249.3\left[\mathrm{~d}, J_{\mathrm{PC}}=5, \mathrm{CO}\right], 108.8\left[\mathrm{~s}, \mathrm{C}\left(\mathrm{C}_{6} \mathrm{H}_{3}\right)\right], 96.7$ [d, $\left.J_{\mathrm{PC}}=17, \mathrm{C}\left(\mathrm{C}_{5} \mathrm{H}_{4}\right)\right], 95.5(\mathrm{~s}, \mathrm{Cp}), 89.6,89.1,87.3\left(3 \times \mathrm{x} \mathrm{s} \mathrm{C}_{5} \mathrm{H}_{4}\right), 84.3(\mathrm{~d}$, $\left.J_{\mathrm{PC}}=12, \mathrm{C}_{5} \mathrm{H}_{4}\right), 74.6\left(\mathrm{~s}, \mathrm{C}_{6} \mathrm{H}_{3}\right), 34.5\left[\mathrm{~s}, \mathrm{C}\left({ }^{t} \mathrm{Bu}\right)\right], 31.7\left(\mathrm{~s}, \mathrm{CH}_{3}\right)$. NMR data for the minor isomer: ${ }^{31} \mathrm{P}$ NMR: $\delta-13.8\left(\mathrm{~d}, J_{\mathrm{PH}}=290, \mu-\mathrm{PHC}_{5} \mathrm{H}_{4}\right) .{ }^{1} \mathrm{H}$ NMR: $\delta 5.44$ (s, 5H, Cp) 5.08, 4.97, (2 x m, br, $\left.2 \times 1 \mathrm{H}, \mathrm{C}_{5} \mathrm{H}_{4}\right), 4.85(\mathrm{~d}$, $\left.J_{\mathrm{HP}}=3,3 \mathrm{H}, \mathrm{C}_{6} \mathrm{H}_{3}\right), 4.83\left(\mathrm{~m}, \mathrm{br}, 2 \mathrm{H}, \mathrm{C}_{5} \mathrm{H}_{4}\right), 3.87\left(\mathrm{dd}, J_{\mathrm{HH}}=1, J_{\mathrm{PH}}=290\right.$, $1 \mathrm{H}, \mathrm{H}-\mathrm{P}), 1.20\left(\mathrm{~s}, 27 \mathrm{H},{ }^{t} \mathrm{Bu}\right) ;{ }^{13} \mathrm{C}\left\{{ }^{1} \mathrm{H}\right\}$ NMR: $\delta 109.5$ [s, $\left.\mathrm{C}\left(\mathrm{C}_{6} \mathrm{H}_{3}\right)\right], 95.2$ (s, Cp), 88.6, 88.5, 87.7, $79.2\left(4 \times \mathrm{s}_{5} \mathrm{C}_{5} \mathrm{H}_{4}\right), 73.1\left(\mathrm{~s}, \mathrm{C}_{6} \mathrm{H}_{3}\right), 35.1$ [s, $\left.\mathrm{C}\left({ }^{t} \mathrm{Bu}\right)\right], 31.5\left(\mathrm{~s}, \mathrm{CH}_{3}\right)$; the resonances for the carbonyls and the phosphorus-bound $\mathrm{C}$ atom in this minor isomer could not be unambiguously identified in the spectrum of the mixture of isomers . Ratio of isomers $=5 / 2$.

Preparation of $\quad\left[\mathrm{FeMo}_{2} \mathrm{Cp}\left(\mu-\boldsymbol{\kappa}^{1}: \boldsymbol{\kappa}^{1}: \boldsymbol{\kappa}^{1}, \boldsymbol{\eta}^{5}-\mathrm{PC}_{5} \mathrm{H}_{4}\right)\left(\eta^{6}-\right.\right.$ $\left.\left.\mathbf{C}_{6} \mathbf{H}_{3}{ }^{\mathrm{t}} \mathbf{B u}_{3}\right)(\mathbf{C O})_{6}\right]$ (5). Solid $\left[\mathrm{Fe}_{2}(\mathrm{CO})_{9}\right](0.025 \mathrm{~g}, 0.069 \mathrm{mmol})$ was added to a tetrahydrofuran solution $(5 \mathrm{~mL})$ of compound $2(0.027 \mathrm{~g}, 0.041$ $\mathrm{mmol}$ ) at $213 \mathrm{~K}$, and the mixture was stirred while allowing the temperature to reach $273 \mathrm{~K}$. After $6 \mathrm{~h}$ stirring at that temperature the solvent and all volatile substances were removed under vacuum, the residue was extracted with petroleum ether and the extracts were chromatographed on an alumina column (activity IV)_at $263 \mathrm{~K}$. Elution with dichloromethane-petroleum ether (1:4) gave a yellow-green fraction yielding, after removal of solvents under vacuum, compound $\mathbf{5}$ as a brown-yellow microcrystalline solid $(0.028 \mathrm{~g}, 82 \%)$. The crystals used in the X-ray study of 5 were grown by slow diffusion at $253 \mathrm{~K}$ of a layer of petroleum ether into a concentrated solution of the complex in toluene. 
Anal. Calcd for $\mathrm{C}_{34} \mathrm{H}_{39} \mathrm{Mo}_{2} \mathrm{FeO}_{6} \mathrm{P}: \mathrm{C}, 49.66 ; \mathrm{H}, 4.78$. Found: C, 49.53; H, 4.70. v(CO) (petroleum ether): 2056 (vs), 2002 (m), 1985 (s), 1976 (vs), 1927 (vs), 1860 (m). ${ }^{31} \mathrm{P}\left\{{ }^{1} \mathrm{H}\right\}$ NMR: $\delta 205.5$ (s, $\left.\mu-\mathrm{PC}_{5} \mathrm{H}_{4}\right) .{ }^{1} \mathrm{H}$ NMR $\left(\mathrm{C}_{6} \mathrm{D}_{6}\right): \delta 5.02(\mathrm{~s}, 5 \mathrm{H}, \mathrm{Cp}), 4.87\left(\mathrm{~d}, J_{\mathrm{HP}}=4,3 \mathrm{H}, \mathrm{C}_{6} \mathrm{H}_{3}\right), 4.81(\mathrm{br}, 1 \mathrm{H}$, $\left.\mathrm{C}_{5} \mathrm{H}_{4}\right), 4.48$ (br, $\left.2 \mathrm{H}, \mathrm{C}_{5} \mathrm{H}_{4}\right), 4.38$ (br, $\left.1 \mathrm{H}, \mathrm{C}_{5} \mathrm{H}_{4}\right), 1.08\left(\mathrm{~s}, 27 \mathrm{H},{ }^{t} \mathrm{Bu}\right)$.

Preparation of $\left[\mathrm{Co}_{2} \mathrm{Mo}_{2} \mathrm{Cp}\left(\mu-\kappa^{1}: \boldsymbol{\kappa}^{1}: \boldsymbol{\kappa}^{1}: \boldsymbol{\kappa}^{1}, \eta^{5}-\mathrm{PC}_{5} \mathrm{H}_{4}\right)(\mathrm{CO})_{7}\left(\eta^{6}-\right.\right.$ $\left.\left.\mathbf{C}_{6} \mathbf{H}_{3}{ }^{\mathrm{t}} \mathbf{B u}_{3}\right)\right](\mathbf{6})$. Solid $\left[\mathrm{Co}_{2}(\mathrm{CO})_{8}\right](0.050 \mathrm{~g}, 0.146 \mathrm{mmol})$ was added to a solution of compound $2(0.030 \mathrm{~g}, 0.046 \mathrm{mmol})$ in dichloromethane (3 $\mathrm{mL}$ ) at room temperature, and the mixture was stirred for $1 \mathrm{~min}$ to give a yellow-brown solution. The solvent was then removed under vacuum and the residue was chromatographed on an alumina column (activity IV) refrigerated with water. Elution with dichloromethane-petroleum ether (1:2) gave a brown fraction yielding, after removal of solvents under vacuum, compound $\mathbf{6}$ as a yellow-brown microcrystalline solid $(0.027 \mathrm{~g}$, $70 \%$ ). The crystals used in the X-ray study of $\mathbf{6}$ were grown by slow diffusion at $253 \mathrm{~K}$ of a layer of petroleum ether (having a few drops of toluene) into a concentrated solution of the complex in dichloromethane. Unfortunately, no satisfactory elemental analysis could be obtained for this product due to its rapid decomposition upon manipulation in the air. v(CO) $\left(\mathrm{CH}_{2} \mathrm{Cl}_{2}\right) 2039$ (vs), 1997 (vs, br), 1976 (m, sh), 1942 (w), 1918 (w, sh), 1765 (w, br) $\mathrm{cm}^{-1} .{ }^{31} \mathrm{P}\left\{{ }^{1} \mathrm{H}\right\} \operatorname{NMR}(243 \mathrm{~K}): \delta 256.5\left(\mathrm{br}, \mu-\mathrm{PC}_{5} \mathrm{H}_{4}\right)$. ${ }^{1} \mathrm{H}$ NMR (243K): $\delta 5.49$ (br, 1H, $\left.\mathrm{C}_{5} \mathrm{H}_{4}\right), 5.44$ (s, br, 5H, Cp), 5.09 (br, $\left.4 \mathrm{H}, \mathrm{C}_{5} \mathrm{H}_{4}, \mathrm{C}_{6} \mathrm{H}_{3}\right), 3.88\left(\mathrm{br}, 1 \mathrm{H}, \mathrm{C}_{5} \mathrm{H}_{4}\right), 3.61\left(\mathrm{br}, 1 \mathrm{H}, \mathrm{C}_{5} \mathrm{H}_{4}\right), 1.27(\mathrm{~s}, 27 \mathrm{H}$, $\left.{ }^{t} \mathrm{Bu}\right) .{ }^{13} \mathrm{C}\left\{{ }^{1} \mathrm{H}\right\}$ NMR $(243 \mathrm{~K}): \delta 265.9,229.4,209.4$ [3 x m, vbr, CO], 109.8 [s, $\left.\mathrm{C}\left(\mathrm{C}_{6} \mathrm{H}_{3}\right)\right], 92.9,92.1\left(2 \times \mathrm{s}, 2 \mathrm{x} \mathrm{C}_{5} \mathrm{H}_{4}\right), 91.7(\mathrm{~s}, \mathrm{Cp}), 87.9(\mathrm{~s}, 2 \mathrm{x}$ $\left.\mathrm{C}_{5} \mathrm{H}_{4}\right), 77.5\left(\mathrm{~s}, \mathrm{C}_{6} \mathrm{H}_{3}\right), 34.8\left[\mathrm{~s}, \mathrm{C}\left({ }^{\mathrm{t}} \mathrm{Bu}\right)\right], 31.6\left[\mathrm{~s}, \mathrm{CH}_{3}\right] \mathrm{ppm}$. The resonance for the P-bound cyclopentadienylidene carbon could not be located.

X-ray Structure Determination for Compound 2. X-ray intensity data were collected on a Nonius FR591-KappaCCD2000 diffractometer using $\mathrm{Cu}-\mathrm{K} \alpha$ radiation at $120 \mathrm{~K}$. Cell dimensions and orientation matrixes were initially determined from least-squares refinements on reflections measured in 3 sets of 30 exposures collected in 3 diferent $\omega$ regions and eventually refined against all reflections. The data collection strategy was calculated with the program Collect. ${ }^{3}$ Data reduction and cell refinement were performed with the programs HKL, Denzo and Scalepack. ${ }^{4}$ The collected frames were then processed for integration by the software SAINT, ${ }^{5}$ and a multi-scan absorption correction was applied with SADABS. ${ }^{6}$ Using the program suite WinGX, ${ }^{7}$ the structure was solved by Patterson interpretation and phase expansion, and refined with full-matrix least squares on $F^{2}$ with SHELXL97. ${ }^{8}$ All non-hydrogen atoms were refined anisotropically and all hydrogen atoms were fixed at calculated geometric positions and given an overall isotropic thermal parameter. Further crystallographic data and structure refinement details for compound $\mathbf{2}$ are given in the CIF file format.

X-ray Structure Determination for Compound 3. X-ray intensity data were collected on a Smart-CCD-1000 BRUKER diffractometer using graphite-monochromated $\mathrm{Mo}-\mathrm{K}_{\alpha}$ radiation at $100 \mathrm{~K}$. The software SMART $^{5}$ was used for collecting frames with omega scans measurement method. The software SAINT was used for the data reduction, ${ }^{5}$ and a multi-scan absorption correction was applied with SADABS. ${ }^{6}$ Using the program suite $\mathrm{WinGX}^{7}$ the structure was solved by Patterson interpretation and phase expansion, and refined with full-matrix least squares on $F^{2}$ with SHELXL97. ${ }^{8}$ All non-hydrogen atoms were refined anisotropically and all hydrogen atoms were fixed at calculated geometric positions and given an overall isotropic thermal parameter. Further crystallographic data and structure refinement details for compound $\mathbf{3}$ are given in the CIF file format.

X-ray Structure Determination for Compound 5. X-ray diffraction intensity data were collected at $150(2) \mathrm{K}$ on a Nonius KappaCCD single crystal diffractometer, using $\mathrm{Cu}-\mathrm{K} \alpha$ radiation $(\lambda=1.5418 \AA)$. Images were collected at a $29 \mathrm{~mm}$ fixed crystal-detector distance, using the oscillation method, with $2^{\circ}$ oscillation and $40 \mathrm{~s}$ exposure time per image. Data collection strategy was calculated with the program Collect. ${ }^{3}$ Data reduction and cell refinement were performed with the programs HKL,
Denzo and Scalepack. ${ }^{4}$ A semi-empirical absorption correction was applied using the program SORTAV. ${ }^{9}$ Using the program suite WinGX, ${ }^{7}$ the structure was solved by Patterson interpretation and phase expansion, and refined with full-matrix least squares on $F^{2}$ with SHELXL97. ${ }^{8}$ All non-hydrogen atoms were refined anisotropically and all hydrogen atoms were fixed at calculated geometric positions and given an overall isotropic thermal parameter. Further crystallographic data and structure refinement details for compound $\mathbf{5}$ are given in the CIF file format.

$\mathrm{X}$-ray Structure Determination for Compound $\mathbf{6} \cdot \mathrm{CH}_{2} \mathrm{Cl}_{2}$. X-ray diffraction intensity data were collected at $100(2) \mathrm{K}$ on a Oxford Diffraction Xcalibur Nova single crystal diffractometer, using $\mathrm{Cu}-\mathrm{K} \alpha$ radiation $(\lambda=1.5418 \AA)$. Images were collected at a $60 \mathrm{~mm}$ fixed crystaldetector distance, using the oscillation method, with $1^{\circ}$ oscillation and variable exposure time per image (4-15 s). Data collection strategy was calculated with the program CrysAlis Pro CCD (Oxford Diffraction Ltd., 2006). ${ }^{10}$ Data reduction and cell refinement was performed with the program CrysAlis Pro. ${ }^{10}$ An empirical absorption correction was applied using the SCALE3 ABSPACK algorithm as implemented in the above program. Using the program suite $\mathrm{WinGX}^{7}$, the structure was solved by Patterson interpretation and phase expansion, and refined with full-matrix least squares on $F^{2}$ with SHELXL97. ${ }^{8}$ All non-hydrogen atoms were refined anisotropically and all hydrogen atoms were fixed at calculated geometric positions and given an overall isotropic thermal parameter. The complex crystallizes with a dichloromethane molecule which was satisfactorily refined. Further crystallographic data and structure refinement details for compound $\mathbf{6}$ are given in the CIF file format.

\section{References}

(1) Armarego, W. L. F.; Chai, C. Purification of Laboratory Chemicals, 5th Edition; Butterworth-Heinemann: Oxford, UK, 2003.

(2) Amor, I.; García, M. E.; Ruiz, M. A.; Saéz, D.; Hamidov, H.; Jeffery, J. C. Organometallics 2006, 25, 4857-4869.

(3) Collect; Nonius BV: Delft, The Netherlands, 1997-2000.

(4) Otwinowski, Z.; Minor, W. Methods Enzymol. 1997, 276, 307.

(5) SMART \& SAINT Software Reference Manuals, Version 5.051 (Windows NT Version); Bruker Analytical X-ray Instruments: Madison WI, 1998.

(6) Sheldrick, G. M. SADABS, Program for Empirical Absorption Correction; University of Göttingen: Göttingen, Germany, 1996.

(7) Farrugia, L. J. J. Appl. Cryst. 1999, 32, 837.

(8) Sheldrick, G. M. SHELXL97: Program for the Refinement of Crystal Structures; University of Göttingen: Göttingen, Germany, 1997.

(9) Blessing, R. H. Acta Crystallogr, 1995, A51, 33-38.

(10) CrysAlis Pro, Oxford Diffraction Limited, Ltd., 2006. 


\section{DFT Analysis of $\left[\mathrm{Mo}_{2} \mathrm{Cp}\left(\mu-\kappa^{1}: \kappa^{1}, \eta^{5}-\mathrm{PC}_{5} \mathrm{H}_{4}\right)(\mathrm{CO})_{2}\left(\eta^{6}-\mathrm{HR}^{*}\right)\right]$.}

Density functional calculations were carried out using the GAUSSIAN03 package, ${ }^{1}$ in which the hybrid method B3LYP was applied with the Becke three parameters exchange functional $^{2}$ and the Lee-Yang-Parr correlation functional. ${ }^{3}$ For the Mo atom a valence double- $\zeta$ quality basis set and LANL2DZ effective core potentials were used. ${ }^{4}$ The light elements ( $\mathrm{P}, \mathrm{O}, \mathrm{C}$ and $\mathrm{H}$ ) were described with $6-31 \mathrm{G}^{*}{ }^{5}$ Geometry optimization were performed under no symmetry restrictions, using initial coordinates derived from X-ray data, and frequency analysis were performed to ensure that a minimum structure is achieved with no imaginary frequencies. Molecular orbitals and frequencies were visualized using Molekel program. ${ }^{6}$

\section{Optimized geometry.}

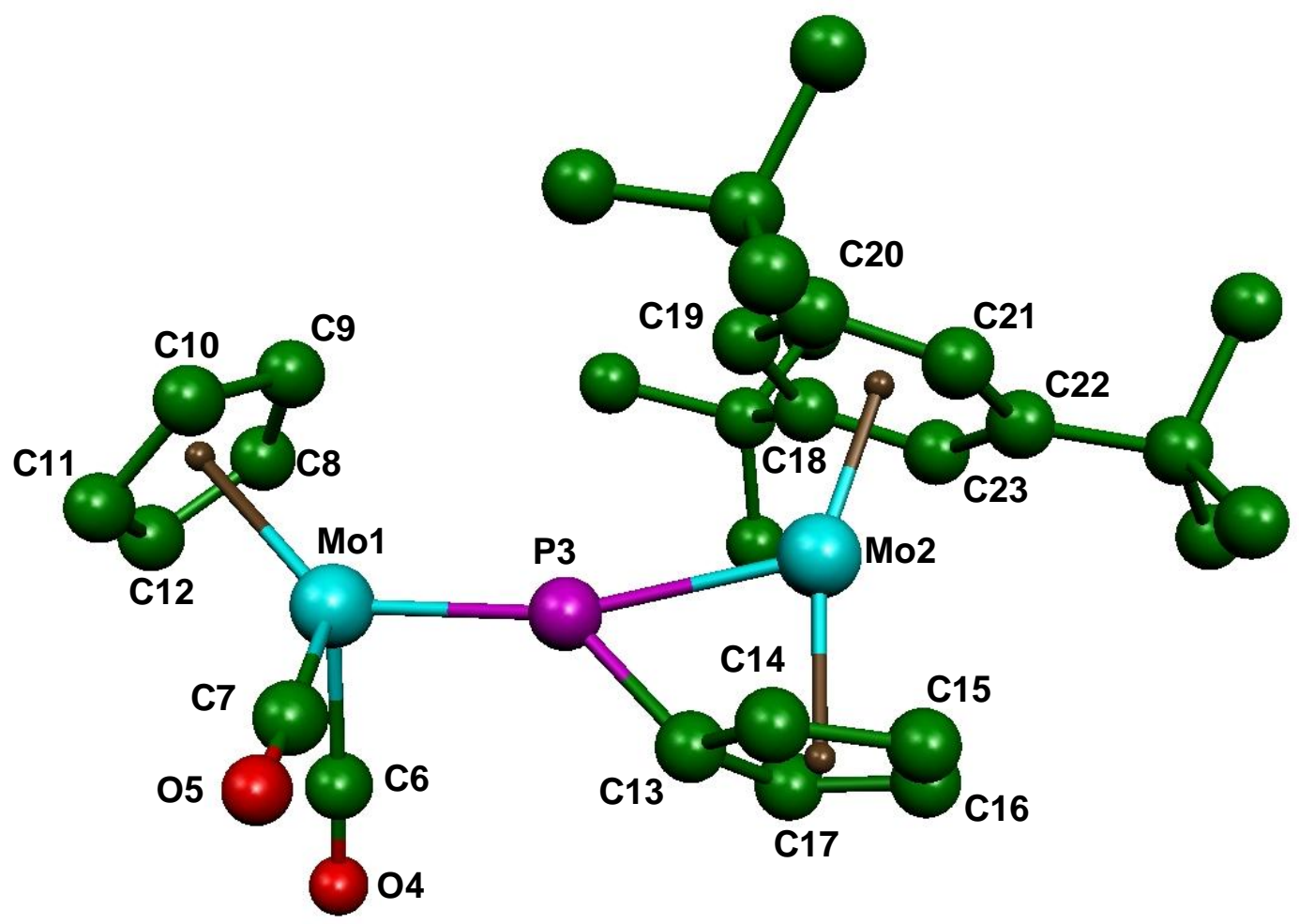

Figure 1 
Table 1: X-ray Structural Data and DFT-Optimized Geometries for $\left[\mathrm{Mo}_{2} \mathrm{Cp}\left(\mu-\kappa^{1}: \kappa^{1}, \eta^{5}-\right.\right.$ $\left.\left.\mathrm{PC}_{5} \mathrm{H}_{4}\right)(\mathrm{CO})_{2}\left(\eta^{6}-\mathrm{HR}^{*}\right)\right]$ (distances in $\AA$, angles in deg.).

\begin{tabular}{cccc}
\hline Parameter & Optimized & X-Ray & Difference \\
\hline $\mathrm{Mo}(1)-\mathrm{P}(3)$ & 2.272 & $2.2518(15)$ & 0.020 \\
$\mathrm{Mo}(2)-\mathrm{P}(3)$ & 2.505 & $2.4027(14)$ & 0.102 \\
$\mathrm{Mo}(1)-\mathrm{C}(7)$ & 1.971 & $1.948(6)$ & 0.023 \\
$\mathrm{C}(7)-\mathrm{O}(5)$ & 1.165 & $1.150(8)$ & 0.015 \\
$\mathrm{Mo}(1)-\mathrm{C}(6)$ & 1.975 & $1.958(8)$ & 0.017 \\
$\mathrm{C}(6)-\mathrm{O}(4)$ & 1.165 & $1.158(2)$ & 0.007 \\
$\mathrm{P}(3)-\mathrm{C}(13)$ & 1.807 & $1.789(6)$ & 0.018 \\
$\mathrm{Mo}(2)-\mathrm{C}(13)$ & 2.231 & $2.208(5)$ & 0.023 \\
$\mathrm{Mo}(2)-\mathrm{C}(18)$ & 2.356 & $2.304(4)$ & 0.052 \\
$\mathrm{Mo}(2)-\mathrm{C}(19)$ & 2.340 & $2.310(5)$ & 0.030 \\
$\mathrm{Mo}(2)-\mathrm{C}(20)$ & 2.354 & $2.324(5)$ & 0.030 \\
$\mathrm{Mo}(2)-\mathrm{C}(21)$ & 2.308 & $2.280(5)$ & 0.028 \\
$\mathrm{Mo}(2)-\mathrm{C}(22)$ & 2.399 & $2.357(5)$ & 0.042 \\
$\mathrm{Mo}(2)-\mathrm{C}(23)$ & 2.391 & $2.285(4)$ & 0.106 \\
$\mathrm{Mo}(2)-\mathrm{C}(14)$ & 2.335 & $2.288(5)$ & 0.047 \\
$\mathrm{Mo}(2)-\mathrm{C}(15)$ & 2.400 & $2.338(5)$ & 0.062 \\
$\mathrm{Mo}(2)-\mathrm{C}(16)$ & 2.391 & $2.335(6)$ & 0.056 \\
$\mathrm{Mo}(2)-\mathrm{C}(17)$ & 2.323 & $2.262(6)$ & 0.061 \\
$\mathrm{Mo}(1)-\mathrm{P}(3)-\mathrm{Mo} 2)$ & 165.2 & $163.73(8)$ & 1.5 \\
$\mathrm{Mo}(2)-\mathrm{P}(3)-\mathrm{C}(13)$ & 59.7 & $61.35(16)$ & -1.7 \\
$\mathrm{C}(7)-\mathrm{Mo}(1)-\mathrm{C} 6)$ & 83.6 & $86.0(3)$ & -2.4 \\
$\mathrm{Mo}(1)-\mathrm{P}(3)-\mathrm{C}(13)$ & 134.8 & $134.91(17)$ & -0.1 \\
\hline
\end{tabular}


Table 2: KS Molecular orbitals of $\left[\mathrm{Mo}_{2} \mathrm{Cp}\left(\mu-\kappa^{1}: \kappa^{1}, \eta^{5}-\mathrm{PC}_{5} \mathrm{H}_{4}\right)(\mathrm{CO})_{2}\left(\eta^{6}-\mathrm{HR}^{*}\right)\right]$.

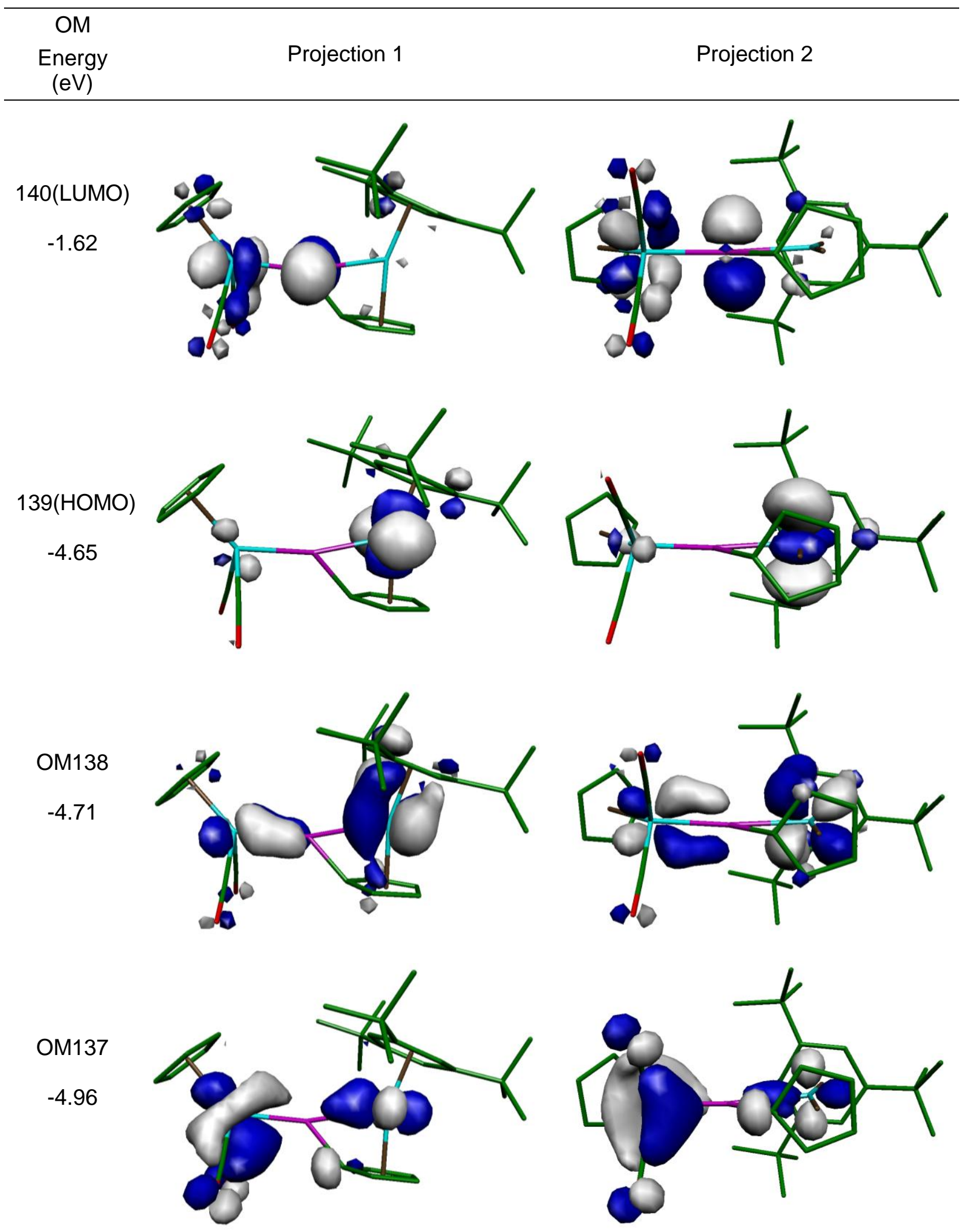


OM136

$-5.09$
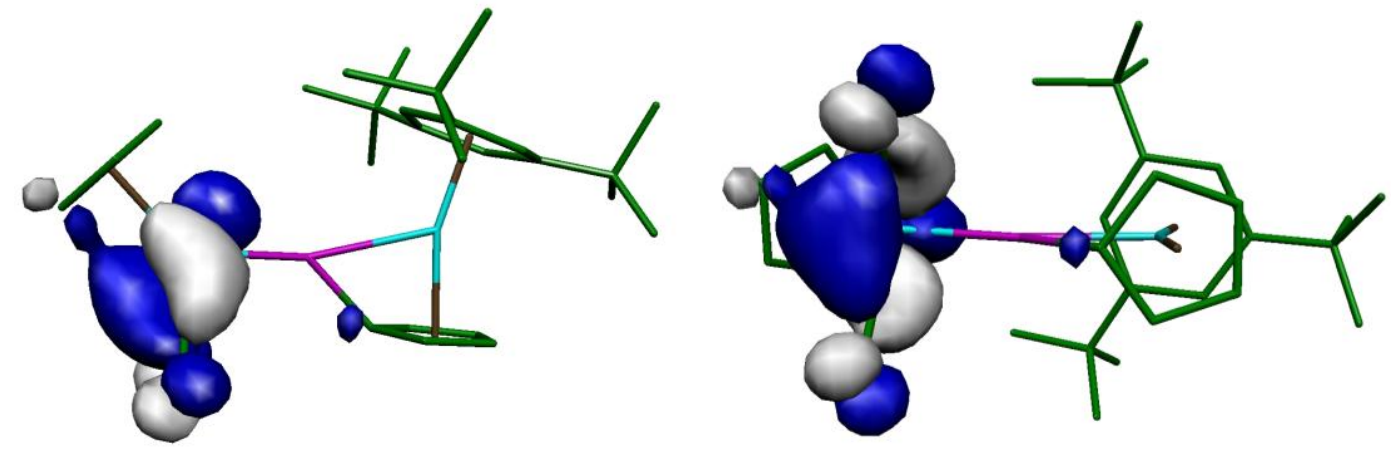

OM135

$-6.05$
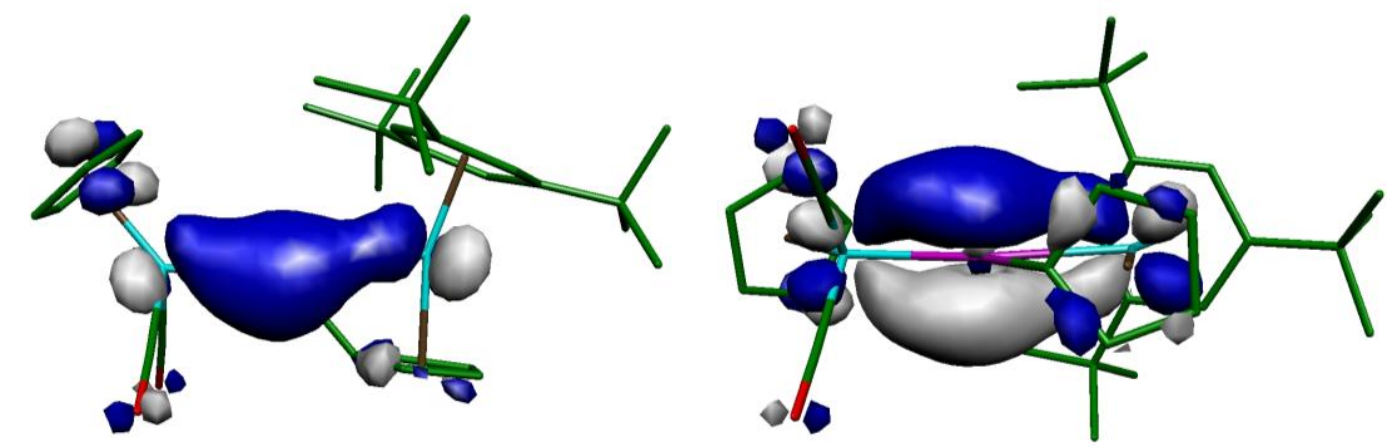

OM134

$-6.49$
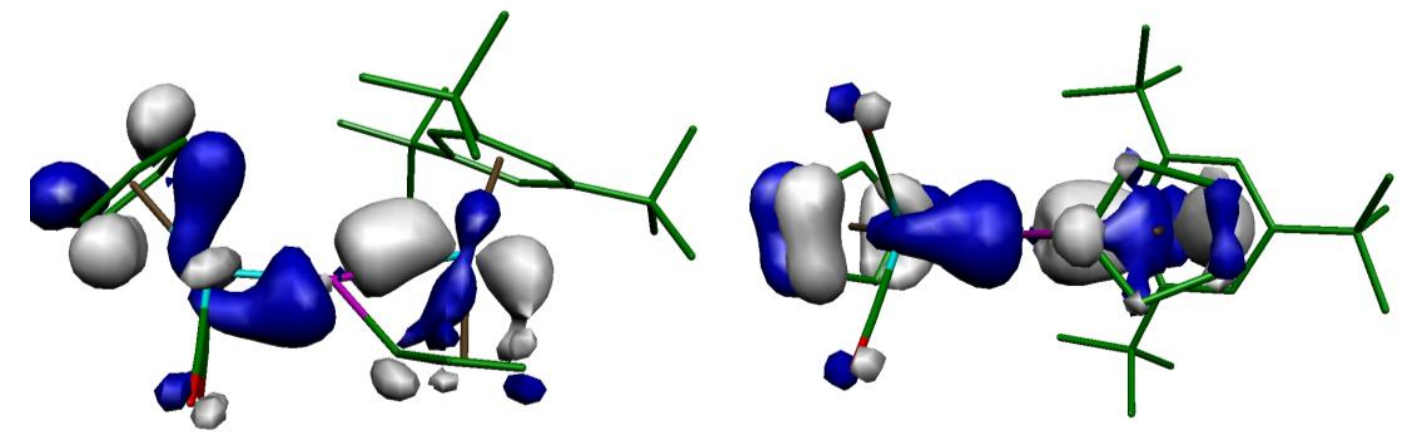
Table 2: Cartesian coordinates for the optimized geometry of $\left[\mathrm{Mo}_{2} \mathrm{Cp}\left(\mu-\kappa^{1}: \kappa^{1}, \eta^{5}-\right.\right.$ $\left.\left.\mathrm{PC}_{5} \mathrm{H}_{4}\right)(\mathrm{CO})_{2}\left(\eta^{6}-\mathrm{HR}^{*}\right)\right]$.

\begin{tabular}{|c|c|c|c|}
\hline & 0.000 & 00 & 0.000 \\
\hline & -0.061782521 & 0.638178840 & 4.69347608 \\
\hline & 0.000000000 & 0.000000000 & 2.271596833 \\
\hline & 1.878697201 & -2.513214292 & \\
\hline & 73 & 3048 & 3891 \\
\hline & 1.171760926 & -1.589684317 & -0.029772829 \\
\hline & 55 & 34 & 0 \\
\hline & 1.2 & 1.7 & -1. \\
\hline & -0.000000000 & 6 & 746 \\
\hline & 1.078424145 & 1.707162850 & -1.2 \\
\hline & 9 & 24 & -2 \\
\hline & 0.894 & 0.83 & -2.1 \\
\hline & 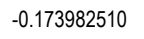 & 42 & 318 \\
\hline & 31 & -1.23 & 4.3 \\
\hline & -1.03 & -1.31 & 5.6 \\
\hline & 75 & -1.4 & 5.7 \\
\hline & 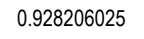 & -1.451825998 & 3709 \\
\hline & 1. & 2.525767875 & 4. \\
\hline & 0.076 & 2.837 & $3.9 c$ \\
\hline & 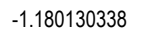 & 4 & 4.565877918 \\
\hline & & & \\
\hline & 0.09 & 2.0 & \\
\hline & 1.303103509 & 2.09 & 5.8 \\
\hline & 2. & 2.824062082 & 3.842906784 \\
\hline & 2. & 9 & \\
\hline & 3.664 & 1.63 & 3.9 \\
\hline & 3.296466778 & 4.06 & 4.5 \\
\hline & - & 3.19 & 3.956104516 \\
\hline & & & \\
\hline & -2.87 & 4.53 & 4.6 \\
\hline & -2.396 & 3.42 & 2.43 \\
\hline & r & & \\
\hline & 1.4600 & & \\
\hline & -0.172 & 3.23 & 8.7 \\
\hline & -0.988 & 0.8 & 8. \\
\hline & 2.219289356 & & \\
\hline & -0. & 6 & \\
\hline & -2.131414482 & 1.919094247 & -1.16 \\
\hline & . & 36 & 2 \\
\hline & & & 0 \\
\hline & -2.397256926 & -1.138 & 3.9 \\
\hline & -1.725288614 & -1.299458556 & 6.5 \\
\hline & 0497 & 1500 & \\
\hline & 1.9720429 & 2 & 4.204224732 \\
\hline & 0.07336 & 1 & .86 \\
\hline & 20 & 2.171847919 & 6.473 \\
\hline & 2.23 & 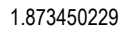 & 6.365892770 \\
\hline & 2.0 & 0 & 30 \\
\hline & 3.535 & & \\
\hline & 1.951720793 & 4.044529373 & \\
\hline & 8492 & 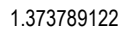 & 5.036396428 \\
\hline & 4.632 & 1.88 & 3.5 \\
\hline & (w) & 0.749775682 & \\
\hline & .6251572 & .93189 & 7.700001 \\
\hline & 52313 & .3388632 & 4.075726 \\
\hline
\end{tabular}

H $\quad 3.481502742 \quad 3.885814497 \quad 5.605263898$

H $\quad-3.399926970 \quad 1.214172428 \quad 3.725466419$

H $\quad-4.577971495 \quad 2.546467127 \quad 3.772158057$

H $\quad-3.819205151 \quad 1.987631566 \quad 5.263445323$

H $\quad-3.016913828 \quad 4.418549086 \quad 5.711523112$

H $\quad-3.807238759 \quad 4.932031209 \quad 4.210714587$

H $\quad-2.088430799 \quad 5.283111076 \quad 4.474824989$

H $\quad-1.689379764 \quad 4.222630379 \quad 2.192943195$

H $\quad-3.372671529 \quad 3.722669864 \quad 2.041547661$

H $\quad-2.087852508 \quad 2.508709964 \quad 1.914764351$

H $\quad 2.262828984 \quad 2.060486819 \quad 8.511945457$

H $\quad 1.751187487 \quad 0.390438698 \quad 8.194507964$

H $\quad 1.395136144 \quad 1.155912544 \quad 9.750881163$

$\begin{array}{llll}H & -0.139183109 & 3.165434017 & 9.875148010\end{array}$

H $\quad-1.157921065 \quad 3.614830327 \quad 8.494456406$

H $\quad 0.578556337 \quad 3.964158818 \quad 8.461938749$

$\begin{array}{llll}H & -0.784391032 & -0.158892584 & 8.296641407\end{array}$

H $\quad-1.990246936 \quad 1.139008786 \quad 8.294161240$

H $\quad-1.009786231 \quad 0.850032674 \quad 9.731101004$ 


\section{References}

(1) Gaussian 03, Revision B.02, Frisch, M. J.; Trucks, G. W.; Schlegel, H. B.; Scuseria, G. E.; Robb, M. A.; Cheeseman, J. R.; Montgomery, Jr., J. A.; Vreven, T.; Kudin, K. N.; Burant, J. C.; Millam, J. M.; Iyengar, S. S.; Tomasi, J.; Barone, V.; Mennucci, B.; Cossi, M.; Scalmani, G.; Rega, N.; Petersson, G. A.; Nakatsuji, H.; Hada, M.; Ehara, M.; Toyota, K.; Fukuda, R.; Hasegawa, J.; Ishida, M.; Nakajima, T.; Honda, Y.; Kitao, O.; Nakai, H.; Klene, M.; Li, X.; Knox, J. E.; Hratchian, H. P.; Cross, J. B.; Bakken, V.; Adamo, C.; Jaramillo, J.; Gomperts, R.; Stratmann, R. E.; Yazyev, O.; Austin, A. J.; Cammi, R.; Pomelli, C.; Ochterski, J. W.; Ayala, P. Y.; Morokuma, K.; Voth, G. A.; Salvador, P.; Dannenberg, J. J.; Zakrzewski, V. G.; Dapprich, S.; Daniels, A. D.; Strain, M. C.; Farkas, O.; Malick, D. K.; Rabuck, A. D.; Raghavachari, K.; Foresman, J. B.; Ortiz, J. V.; Cui, Q.; Baboul, A. G.; Clifford, S.; Cioslowski, J.; Stefanov, B. B.; Liu, G.; Liashenko, A.; Piskorz, P.; Komaromi, I.; Martin, R. L.; Fox, D. J.; Keith, T.; Al-Laham, M. A.; Peng, C. Y.; Nanayakkara, A.; Challacombe, M.; Gill, P. M. W.; Johnson, B.; Chen, W.; Wong, M. W.; Gonzalez, C.; and Pople, J. A.; Gaussian, Inc., Wallingford CT, 2004.

(2) Becke, A. D. J. Chem. Phys. 1993, 98, 5648.

(3) Lee, C.; Yang, W.; Parr, R. G. Phys. Rev. B 1988, 37, 785.

(4) Hay, P. J.; Wadt, W. R. J. Chem. Phys. 1985, 82, 299.

(5) (a) Hariharan, P. C.; Pople, J. A. Theor. Chim. Acta 1973, 28, 213. (b) Petersson, G. A.; Al-Laham, M. A. J. Chem. Phys. 1991, 94, 6081. (c) Petersson, G. A.; Bennett, A.; Tensfeldt, T. G.; Al-Laham, M. A.; Shirley, W. A.; Mantzaris, J. J. Chem. Phys. 1988, 89, 2193.

(6) Portmann, S.; Lüthi, H. P.; MOLEKEL: An Interactive Molecular Graphics Tool. CHIMIA 2000, 54, 766. 